\title{
Mutual Coexistence in WBANs: Impact of Modulation Schemes of the IEEE 802.15.6 Standard
}

\author{
Marwa BOUMAIZ ${ }^{1}$, Mohammed EL GHAZI ${ }^{2}$, Mohammed FATTAH ${ }^{3}$ \\ Anas BOUAYAD ${ }^{4}$, Moulhime EL BEKKALI ${ }^{5}$ \\ Laboratory of Artificial Intelligence, Data Sciences and Emerging Systems \\ Sidi Mohammed Ben Abdellah University, FEZ, Morocco ${ }^{1,2,4,5}$ \\ EST, Moulay Ismail University, Meknes, Morocco ${ }^{3}$
}

\begin{abstract}
Due to the mobility of subjects carrying wireless Body Area Networks (WBANs), a BAN may be found in an environment that contains other adjacent BANs, which may influence its proper functioning. The purpose of this paper is to study the effect of interference between adjacent BANs on the performance of a reference $\mathrm{BAN}$ in terms of packet loss rate (PLR), while considering the following four parameters: the distance separating adjacent BANs, the number of nodes and traffic payload of an interferer BAN, and the transmission data rate. The study is conducted for the two modulation schemes proposed by the IEEE 802.15.6 standard in the $2.4 \mathrm{GHz}$ narrow band, which are: Differential Binary Phase Shift Keying (DBPSK) modulation and Differential Quadrature Phase Shift Keying (DQPSK) modulation. Simulation results have shown that the adoption of a lower-order modulation such as DBPSK can reduce the effect of interference among adjacent BANs.
\end{abstract}

Keywords-Body Area Network (BAN); mutual coexistence; interference; Differential Binary Phase Shift Keying (DBPSK); Differential Quadrature Phase Shift Keying (DQPSK)

\section{INTRODUCTION}

Wireless body area networks, commonly known as BANs, are a novel technology that has emerged with the recent growth of low-power and low-cost microelectronic systems [1]. In the medical field, these systems can be integrated in a wide range of applications [2], as they are able to remotely monitor patients' physiological conditions, and send these data to a nearby coordinator. Equipped with more resources than a simple sensor, the coordinator is able to transfer the information collected by sensors directly to healthcare centers to be analyzed by a medical staff. This remote monitoring not only saves time, but also allows early detection of health problems without invading the patient's privacy or employing full-time medical staff [3].

As a matter of fact, BANs extend over several fields of applications, other than medical ones. They are also present in the fields of cognitive biometry, military, learning and serious gaming, and sports [4]. This variety of applications envisioned for BANs, gave rise to the IEEE 802.15.6 standard [5], that aims to provide an international norm for highly reliable, bodywide, short-range wireless communications, by supporting a broad range of data rates, ranging from $75.9 \mathrm{Kbps}$ (narrowband) to $15.6 \mathrm{Mbps}$ (ultra-wideband). The standard provides a sophisticated MAC layer with three access modes [6] [8] [7], serving three physical layers which are selected according to the intended application. These are the Narrowband (NB), Ultra-Wideband (UWB) and Human Body Communication (HBC) layers. The NarrowBand physical layer alone provides seven different frequency bands, including the 2.4 GHz band (2400-2483.5 MHz), which uses DBPSK and DQPSK to code the useful information. This band is often preferred over the others due to its worldwide availability[7]. It is also the most mature band [8] offering a greater bandwidth. In addition, it is based on well-known PHY components, which are already widely used in WiFi and Bluetooth [9], and is also characterized by the use of small antennas [10], which makes it perfectly adapted to most on body BAN applications.

However, a BAN can be found in environments where several other BANs or wireless technologies (wifi, bluetooth, zigbee...) coexist. This is the case of interference. Therefore, a BAN can face two kinds of interference: Intra-BAN and or inter-BAN interference. In the first type, interference occurs between nodes of the same BAN. However, this can easily be avoided by using time division channel access [11]. As for the second type, interference is either generated by adjacent BANs [12], or other wireless technologies operating at the same frequency band $(2.4 \mathrm{GHz}$ in particular). When multiple adjacent BANs use the same communication channel (the same frequency), interference can take place, as the active superframes may overlap [13]. However, according to the IEEE 802.15.6 standard, packets will be retransmitted in case of interference for a certain period of time, and therefore there is a trade-off to be made between throughput and energy efficiency at the relevant BAN [13].

There are numerous research studies that focus on interference between adjacent BANs, some of which are presented in Section II. In addition, many of them are interested in reducing or cancelling interference by proposing several solutions at both physical and MAC layers. For example, at the physical layer level, power control solutions may be a possible option to maintain link quality [14]. But when the interference level becomes important, these solutions cannot be suitable for WBANs, because if a WBAN coordinator cannot receive signals coming from a sensor node for example, and by applying power control policies, this sensor will increase its transmit power to be able to send its packets correctly. However, by doing this, it is possible to impact eventual adjacent BANs, which in turn will increase their transmission power, making it difficult for any link to operate with an acceptable quality. Therefore, other adaptive

*Corresponding Author 
methods [15], [11] have been proposed which aim to take advantage of other physical layer parameters (modulation, duty cycle, rate, etc.) to reduce interference in BAN networks. However, these parameters must first be studied in different interference scenarios (change in inter-BAN distance, change in bit rates, change in number of nodes, etc.) for a better evaluation.

This research paper evaluates and analyzes the impact of modulation scheme ( $\pi / 2$ DBPSK and $\pi / 4$ DQPSK as described by the IEEE 802.15.6 standard in the $2.4 \mathrm{GHz}$ narrowband) on BAN transmission performance in a mutual interference environment between adjacent BANs. In this manuscript, a detailed study is evaluated by using three scenarios, and three relevant parameters which are the inter-BAN distance (scenario 1), the number of nodes (scenario 2) and the traffic payload (scenario 3) of the interferer BAN. All three scenarios investigate the BAN transmission performance, in terms of packet loss rate (PLR), for three data rate levels (low, medium and high) and two modulation schemes $\pi / 2$ DBPSK and $\pi / 4$ DQPSK.

The remainder of this article is organized as follows: Section II introduces a state of the art on co-channel interference between adjacent BANs. Then, in Section III the three scenarios studying mutual coexistence are defined, along with the adopted configuration at both the physical and mac layer levels. Next, Numerical study results are reported and analyzed in Section IV. Finally, the paper is concluded in Section V.

\section{CO-CHANNEL INTERFERENCES BETWEEN ADJACENT BANS: STATE OF THE ART}

Mutual interference occurs when a BAN's coordinator receives signals from other nodes of other BANs in its vicinity. Interfering BANs are then said to use the same transmission channel, leading to a packet transmission conflict as the active transmission periods overlap [13].

Research in literature dealing with interference between adjacent BANs can be divided into two categories: interference analysis studies and interference mitigation/cancellation studies. In the following, most relevant works in both of the two categories are presented.

\section{A. Interference Analysis Research}

Interference analysis studies can be divided into three subgroups: simulation-based studies, empirical based studies, and analytical modelling based studies [3].

1) Simulation based studies: Using simulations, Wang et al. [16] analyzed mutual interference for the ultra-wideband IEEE 802.15.6 standard. In this work, two types of receivers were considered, "duty-cycled sampling receiver" and "chirp receiver". Authors observed that the "chirp receiver" guarantees better results than the cycled sampling receiver in terms of PLR when the traffic density increases (Ten adjacent users).

2) Empirical based studies: Davenport et al. [17] used a test-bed study and simulations to investigate the effect of interference from adjacent BANs on the PLR. For this purpose, ten persons carrying BANs were considered in a state of movement in an office area. The PLR measurement in this scenario included different communication techniques: listen before talk (LBT), frequency hopping (FH), and automatic repeat request (ARQ) [3]. It was demonstrated in this work that the combination of LBT and ARQ gives better results.

The assessment of interference between adjacent BANs in case of mobility in indoor/outdoor environments was further discussed in [18]. A scenario of five persons moving in an office was considered, highlighting the effect of distance between nodes and their orientations on interference. The findings of this study showed that distance between nodes does not always have a significant impact on interference in mobility conditions, which is not the case for stationary BANs. However, the orientation of the transceivers has a remarkable effect in reducing interference, especially in outdoor environments which experience fewer reflections and multipath effects.

3) Analytical modelling-based studies: Wang et al. [19] proposed a mathematical model for the characterization of adjacent channel interference in BANs based on Gamma distribution. Authors found the minimum distance between sensors that guarantees an acceptable Signal to Interference Ratio (SIR).

Zhang et al. [20] proposed a mathematical approach to model the average SIR and the probability of collision in the case of co-channel interference. In this study several access methods were considered, including TDMA (Time Division Multiple Access), FDMA (frequency division multiple access), FH (frequency hopping), and CDMA (code division multiple access). Authors showed that when there is no coordination between adjacent BANs, TDMA, FDMA and FH guarantee a similar performance in terms of PLR and BER, which remains better than CDMA. However, in the presence of coordination between adjacent BANs (synchronization), the FDMA and FH protocols have shown better results in interference cancellation.

\section{B. Interference Mitigation Studies}

Research works aimed at reducing or avoiding interference between adjacent BANs tend to propose the following solutions [3]:

1) Time spacing: Interference mitigation techniques (mutual and cross interference) proposed in this category are essentially based on TDMA. This is to prevent nodes of the same or several BANs, or other networks sharing the same transmission channel, from sending their data simultaneously, which leads to collisions. But they are rather suitable solutions for reducing mutual interference [21], [22], [23], [24], [25], [26]. The concept of these solutions consists in rescheduling data packets to avoid interference, by managing transmissions in empty time slots. However, this process can be quite challenging, as frequent coordination exchanges between adjacent BANs are required to know their schedule, which is a real challenge, given the energy constraint in BAN nodes.

2) Frequency spacing: Frequency spacing solutions make judicious use of the available frequency channels for BANs. 
The solutions reported in this category reduce interference levels by implementing channel allocation algorithms that specifically address multi-channel networks.

As discussed, for example, in [27], [28], [29], [30], where authors proposed some solutions to reduce interference in BANs based on frequency spacing. The common point between these studies is that they all rely on the detected interference level, to assign different channels to nodes, to mitigate the impact of interference. Nevertheless, the problem of insufficient channels can be faced in these solutions, particularly when interference occurs with networks having high-bandwidth channels [3].

3) Code diversity: This category mainly targets CDMAbased BANs. the idea here is to choose orthogonal codes to what is used by other adjacent networks to reduce interference [31], [32], [33], [34]. However, these solutions are sometimes slightly complex in terms of estimating the interference level.

4) Standard modification: This category aims to improve the MAC mechanisms implemented by BAN standards. The following research [35] [36] are examples of interference mitigation studies, that have revised and restructured what is proposed in zigbee low power and IEEE 802.15.6 standards respectively, in order to improve coexistence. Therefore, these solutions cannot be applied until they are formally added to the standards [3].

5) Standards adaptation: Unlike standard modification solutions, the solutions in this category reduce interference by adapting to BAN standards without modifying them [37], [38], [39], [40].

6) Hybrid solutions: Same examples of hybrid solutions to reduce interference in BANs are developed in [41] and [42]. Authors used a combination of the above-mentioned methods to exploit the advantages and reduce the shortcomings of one technique over another.

\section{DESCRIPTION OF THE STUdy ScENARIOS AND CONFIGURATION OF THE PHYSICAL AND MAC LAYERS}

\section{A. The Study Scenarios}

In this study, to evaluate the behavior of modulation schemes in a mutual coexistence environment, two BAN models based on the IEEE 802.15.6 standard are considered. The first one is the reference model, called "R-BAN" in this paper. This is the model on which the transmission performance analysis will be carried out and the second one will act as an interferer BAN named "I-BAN". Both of these models operate in the NB layer at $2.45 \mathrm{GHz}$, using a single hop star topology, in which sensor nodes transmit their data directly to the coordinators with no need for relays [43]. This topology has also been chosen in several studies for its better performance [44].

R-BAN consists of eleven sensor nodes, including a coordinator (or hub) placed toward the center of a patient's belly as shown in Fig. 1. The ten other sensors are distributed over the different parts of the body (positions from A to J). The choice of these node positions is driven by the need to monitor the majority of vital signs described in on-body BAN medical applications for the IEEE 802.15.6 standard [45][46]. As for IBAN, it assumes the same number and node positions in scenarios 1 and 3, while in scenario 2 the number of nodes varies.

1) Scenario 1: varying the distance between $R-B A N$ and I$B A N$ : The purpose of this first scenario is to present the effect of varying the inter-BAN distance [12] on mutual interference at low, medium and high data rates, i.e. $3 \mathrm{kbps}, 44 \mathrm{kbps}$ and 72 kbps respectively, as well as evaluating the behavior of $\pi / 2$ DBPSK and $\pi / 4$ DQPSK modulation schemes in this scenario. For this, the following three cases are considered:

- Case 1: absence of mutual interference: R-BAN operates alone.

- Case 2: presence of I-BAN at $6 \mathrm{~m}$ distance from the reference BAN.

- Case 3: presence of the I-BAN at $3 \mathrm{~m}$ distance from the reference BAN.

In the first case packet loss rate (PLR) at R-BAN is simulated in the absence of mutual interference using both DBPSK and DQPSK modulations. This is for the three mentioned data rates (3kbps, 44kbps and $72 \mathrm{kbps}$ ). In the second case, the interferer BAN is added at $6 \mathrm{~m}$ from the RBAN and then this distance is reduced to $3 \mathrm{~m}$ in the third case, so that the effect of this distance reduction on the performance of R-BAN can be evaluated in terms of PLR at low, medium and high data rates. Fig. 2 shows the three cases of scenario 1 .

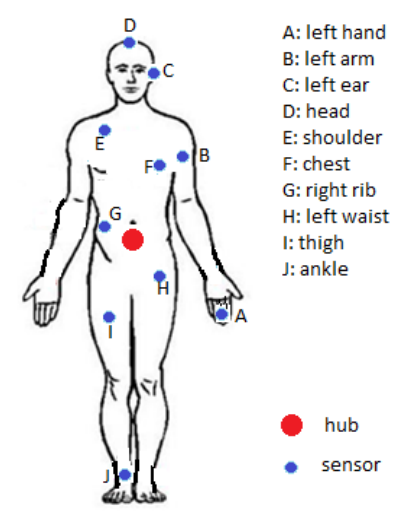

Fig. 1. Reference BAN Model "R-BAN".

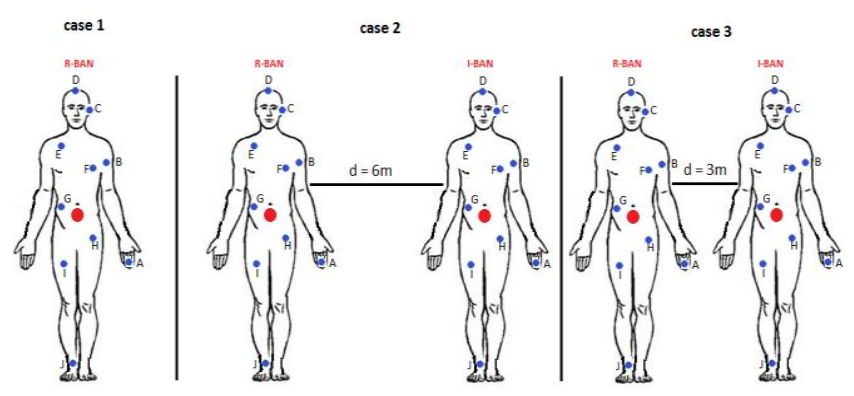

Fig. 2. Scenario 1 Study Cases. 
2) Scenario 2 : Changing the number of nodes of I-BAN: The purpose of this scenario is to evaluate the performance of R-BAN by studying the impact of the number of I-BAN nodes, as well as that of DBPSK and DQPSK modulation schemes acting under these conditions, while fixing the distance between the two BANs at $6 \mathrm{~m}$. The number of nodes in R-BAN is maintained unchanged, to 11 sensors. In this study scenario the following settings for the I-BAN will be examined:

- Case 1: I-BAN with 2 body sensors.

- Case 2: I-BAN with 5 body sensors (one coordinator and four sensor nodes).

- Case 3: I-BAN with 10 body sensors (one coordinator and nine sensor nodes).

- Case 4: I-BAN with 15 body sensors (one coordinator and 14 sensor nodes).

- Case 5: I-BAN with 21 body sensors (one coordinator and 20 sensor nodes).

3) Scenario 3 : Varying the traffic payload of I-BAN: To assess the impact of traffic payload on the performance of the reference BAN, for the case of co-channel interference, the same positions and number of nodes in BANs are kept as in Scenario 1 but varied the traffic payload (in Bytes) of I-BAN, while maintaining the R-BAN payload set at 100 Bytes. The inter-BAN distance is fixed at $6 \mathrm{~m}$ and the behavior of DBPSK and DQPSK modulations is also evaluated in this scenario. For this purpose, the following three cases are taken into account:

- Case 1: I-BAN traffic payload is 10 Bytes.

- Case 2: I-BAN traffic payload is 100 Bytes.

- Case 3: I-BAN traffic payload is 200 Bytes.

\section{B. DBPSK and DQPSK Modulations}

According to the IEEE 802.15.6 standard, in the $2.4 \mathrm{GHz}$ NarrowBand, binary information (superframe payload) is modulated using one of the two following differential phase modulations: $\pi / 2$ DBPSK or $\pi / 4$ DQPSK[47], allowing data to be encoded in the phase of the reference signal. These differential modulations have the advantage of being compatible with non-coherent receivers.

$\pi / 2$ DBPSK modulation uses two relative phases to encode the data $(+\pi / 2$ and $-\pi / 2)$ [48]. For this reason, One-bit symbols are transmitted in the two phases of the same carrier (Asin $(2 \pi \mathrm{ft}))$ but spaced by $180^{\circ}$, as follows:

- For a bit $0 \rightarrow S(t)=A \sin \left(2 \pi f t+90^{\circ}\right)$

- For a bit $1 \rightarrow S(t)=A \sin \left(2 \pi f t-90^{\circ}\right)$

Regarding $\pi / 4 \mathrm{DQPSK}$ modulation, two bits per symbol can be encoded using 4 phases shifted by $\pi / 4$. Moreover, there is always a phase shift even when the adjacent symbols are exactly the same. This results in the following 8 signals:
- For 00 :

$\mathrm{S}(\mathrm{t})=\mathrm{A} \sin (2 \pi \mathrm{ft})$ or $\mathrm{A} \sin (2 \pi \mathrm{ft}+45)$

- For 01 :

$\mathrm{S}(\mathrm{t})=\mathrm{A} \sin (2 \pi \mathrm{ft}+90)$ or $\mathrm{A} \sin (2 \pi \mathrm{ft}+135)$

- For 10 :

$\mathrm{S}(\mathrm{t})=\mathrm{A} \sin (2 \pi \mathrm{ft}+180)$ or $\mathrm{A} \sin (2 \pi \mathrm{ft}+225)$

- For 11 :

$\mathrm{S}(\mathrm{t})=\mathrm{A} \sin (2 \pi \mathrm{ft}+270)$ or $\mathrm{A} \sin (2 \pi \mathrm{ft}+315)$

Among the main differences between the two types of modulations are data rate and robustness against bit errors:

- Data rate: DBPSK allows the transmission of one bit per symbol, instead of two in DQPSK modulation, which means that DQPSK modulation can double the data rate, whilst using the same bandwidth, this is shown in the equation [44] :

$\boldsymbol{R}_{\boldsymbol{d}}=\left(\frac{\boldsymbol{R}_{s} \cdot N}{S} * \frac{k}{n}\right)(\boldsymbol{k b p s})$

Where Rd is the information rate, Rs is the symbol rate, $\mathrm{S}$ is the spreading factor, $\mathrm{k} / \mathrm{n}$ is the $\mathrm{BCH}$ code rate, and $\mathrm{N}$ is a variable related to the modulation order $M$ by $M=2 N$. Therefore, as the modulation order increases, the bit efficiency increases as well.

- Modulation robustness: the constellation diagram of the two modulations shows that the separation between the phases in DBPSK is greater than in DQPSK: the two phases in DBPSK are separated by $180^{\circ}$, whereas in DQPSK the separation is only $45^{\circ}$, which makes DBPSK modulation much more robust against bit errors.

\section{Physical Layer Configuration}

1) On-body channel model : In on-body medical applications, signal propagation takes place on the surface of the human body. In addition to this propagation, which may include a combination of surface waves, creeping waves, diffracted waves, refracted waves and free space propagation waves depending on the position of the antenna [49] [44], Consideration should also be given to the effects of antennahuman body interaction resulting from the placement of body sensors on or near the patient's body, including near-field coupling effects, radiation pattern distortion and antenna impedance changes. These effects impact the functional performance of sensors by degrading their efficiency and reducing the reliability of the collected physiological signals [50]. Due to these issues, a good characterization of the propagation channel is necessary before any design of WBAN solutions.

Assuming that signal propagation in the reference BAN takes place at the body surface, between body sensors, two types of on-body propagation channels can be distinguished: 
- The line of sight on-body channel (LOS): free of any kind of obstruction between each sensor and the coordinator,

- The non-line of sight on-body channel (NLOS) where obstacles may exist in the link of each two communicating nodes.

Therefore, IEEE 802.15.6 standard has defined, for the 2.4 $\mathrm{GHz}$ narrowband, two path loss models, describing propagation with and without line-of-sight, called CM3A and CM3B, respectively [51]. In this work, the CM3B model is adopted as a path loss model. According to this model, path loss decreases exponentially around the perimeter of the body when the BAN nodes are not in line-of-sight. It flattens over large distances due to the addition of multipath components from indoor environments [51]. Path loss in CM3B model is defined by equation 2 :

$P L(d)[d B]=-10 \log _{10}\left(P_{0} e^{-m_{0} d}+P_{1}\right)+\sigma_{P} n_{P}$

Where :

- PL (d) refers to the path loss in $\mathrm{dB}$ at a distance $\mathrm{d}$,

- P0 is a component related to the average losses occurring near the transmitter and depends on the type of antenna.

- P1 is the average attenuation of the components in an indoor environment irradiated from the body and reflected towards the receiving antenna.

- $\mathrm{m} 0$ represents the average exponential decay rate in $\mathrm{dB}$ $/ \mathrm{cm}$ of the creeping wave component diffracting around the body.

- $\mathrm{np}$ is a Gaussian random variable of mean and unit zero.

- $\sigma \mathrm{p}$ is the log-normal variance in $\mathrm{dB}$ around the mean, representing variations measured at different locations in the body and room.

The distances separating each node from the hub were measured and assuming that the proposed network is subject to the same environmental conditions as those of a hospital room, path loss values were also calculated for all the links of the BANs considered. The parameters values of the CM3B model, considered in a hospital room conditions, are presented in Table I [51].

2) Radio module configuration: At the physical layer, a radio chip proposal developed by Alan Wong et al. [52] is considered, which is compatible with the recommendations of the IEEE 802.15.6 standard for the 2.4 GHz NB physical layer. In Table II, the adopted configuration for the radio modules of the adjacent BANs in the three scenarios of the study is presented.

\section{MAC Layer Configuration}

The IEEE 802.15.6 MAC layer provides three access modes to the transmission channel [5]. In this paper, the beacon mode with superframe boundaries is chosen in this mode, the Hub (coordinator node) divides its time axis into several access phases. As a result, the R-BAN model is based on a hybrid MAC layer configuration, combining a scheduled access based on the TDMA, and a contention access based on the CSMA/CA. Thus, a beacon period of 32 allocation intervals of $10 \mathrm{~ms}$ each is defined, i.e. $320 \mathrm{~ms}$ beacon period. If each node of the network is allocated three slots $(30 \mathrm{~ms})$ for scheduled access (TDMA), we end up with 30 allocation slots (300 ms) corresponding to the 10 nodes of the BAN reserved for scheduled access, the two remaining allocation slots (20 ms) will be dedicated to random access (CSMA/CA). The polling mechanism is also activated.

TABLE I. PARAMETERS VALUeS OF CM3B MOdel

\begin{tabular}{|l|l|}
\hline Parameter & Value \\
\hline Frequency $(\mathrm{GHz})$ & 2.45 \\
\hline$P_{0}[\mathrm{~dB}]$ & -25.8 \\
\hline$m_{0}[\mathrm{~dB} / \mathrm{cm}]$ & 2.0 \\
\hline$P_{1}[\mathrm{~dB}]$ & -71.3 \\
\hline$\sigma_{P}[\mathrm{~dB}]$ & 3.6 \\
\hline
\end{tabular}

TABLE II. R-BAN AND I-BAN RADIO MOdULE CONFIGURATION [12][43]

\begin{tabular}{|l|l|l|}
\hline Parameters & R-BAN & I-BAN \\
\hline $\begin{array}{l}\text { Packet transmission } \\
\text { rate (kbps) }\end{array}$ & 3,44 and 72 & 3,44 and 72 \\
\hline Modulation & $\begin{array}{l}\text { DBPSK } \\
\text { DQPSK }\end{array}$ & $\begin{array}{l}\text { DBPSK à 3kbps et } \\
\text { 44kbps. } \\
\text { DQPSK à72kbps. }\end{array}$ \\
\hline hub sensitivity & $\begin{array}{l}-104 \mathrm{dBm} \text { for } \\
\text { DBPSK. } \\
-96.5 \mathrm{dBm} \text { for } \\
\text { DQPSK. }\end{array}$ & $\begin{array}{l}\bullet-104 \mathrm{dBm} \text { for DBPSK. } \\
1 \mathrm{MHz}\end{array}$ \\
\hline Noise bandwidth & $-104 \mathrm{dBm}$ & $1 \mathrm{MHz}$ for DQPSK. \\
\hline Noise floor & $-10 \mathrm{dBm}$ & $-104 \mathrm{dBm}$ \\
\hline Transmit power & $1 \mathrm{~ms}$ & $1 \mathrm{mb}$ \\
\hline $\begin{array}{l}\text { Clear Channel } \\
\text { Assessment Duration }\end{array}$ & -95 & -95 \\
\hline CCA threshold & $5.9 \mathrm{~mW}$ & $5.9 \mathrm{~mW}$ \\
\hline $\begin{array}{l}\text { Power consumption } \\
\text { for Tx mode }\end{array}$ & $0.05 \mathrm{~mW}$ & $0.05 \mathrm{~mW}$ \\
\hline Idle mode supply & $2450 \mathrm{Mhz}$ & $2450 \mathrm{Mhz}$ \\
\hline Frequency & $1.5 \mathrm{~s}$ & $1 \mathrm{~s}$ \\
\hline Startup delay & & \\
\hline
\end{tabular}

\section{NUMERICAL STUDY AND RESUlTS ANALYSIS}

The numerical study of the three study scenarios is carried out using the new version of Castalia simulator (3.3) [53]. This framework is based on OMNeT++(4.6) platform and supports the IEEE 802.15.6 standard, which justifies its use in the numerical study.

In each scenario, packet loss rate (PLR) is evaluated according to the modulation scheme used in R-BAN $(\pi / 2$ DBPSK and $\pi / 4$ DQPSK modulation) at low, medium and high data rate. 
For all performed simulations, R-BAN transmits its packets, for each one of the data rates levels, using successively the two modulation schemes: DBPSK and DQPSK. As for I-BAN, it uses DBPSK for low and medium data rate rates and DQPSK for high data rate.

\section{A. Scenario 1: Effect of DBPSK and DQPSK Modulation Schemes on the Transmission Performance (PLR) of R- $B A N$, as a Function of the Inter-BAN Distance.}

In Fig. 3, 4 and 5, PLR at R-BAN level is presented for low, medium and high data rate respectively, for the three inter-BAN distance cases (case 1: absence of I-BAN, case 2: distance $=6 \mathrm{~m}$, case 3 : distance $=3 \mathrm{~m}$ ). A first visual analysis of the three figures (cases 2 and 3 in the three figures) clearly shows, as expected a priori, that the global transmission performance of the R-BAN network deteriorates (increase of PLR) as the level of mutual interference increases (smaller distance between adjacent BANs). In the absence of I-BAN (case 1, absence of inter-BAN interference) and when R-BAN nodes transmit their packets at a low rate $(3 \mathrm{kbps}$, Fig. 3$)$, a slightly lower packet loss rate $(\mathrm{PLR} \approx 7.63 \%$ ) is noticed with DBPSK modulation than with DQPSK modulation $(\mathrm{PLR} \approx$ $10.81 \%$ ). However, in the presence of I-BAN (cases 2 and 3, inter-BAN interference) and for both of the modulation schemes studied, the PLR increases significantly especially when the inter-BAN distance decreases from $6 \mathrm{~m}$ to $3 \mathrm{~m}$. However, the influence of modulation schemes is inverted this time to designate DQPSK as a very suitable modulation choice for low data rates. Indeed, the results in Fig. 3 show, for an inter-BAN distance of $6 \mathrm{~m}$, a PLR (R-BAN) approaching $73.8 \%$, (DBPSK modulation), which is significantly higher than that obtained with DQPSK modulation (11.63\%). This difference in transmission performance is even more pronounced as the level of inter-BAN interference is high (case 3: inter-BAN distance $=3 \mathrm{~m}$ ). This difference in the performance of the two studied modulations can be related to the difference in sensor sensitivity linked to the adopted modulation scheme. The receiver sensitivity is much lower with DBPSK modulation $(-104 \mathrm{dBm})$ than with DQPSK modulation $(-96.5 \mathrm{dBm})$. This allows the R-BAN coordinator, when configured with DBPSK modulation instead, to pick up even very low-level signals coming from the various nodes of I-BAN. the coexistence between adjacent BANs is more severely affected with high data rate transmissions [3] [4]. Indeed, it is noticed (Fig. 5) that the behavior of the modulation schemes changes compared to the case of low and medium data rate transmissions (Fig. 3 and 4). This time, it is rather the DBPSK modulation that gives a lower PLR than in DQPSK. This can be explained by the robustness of DBPSK modulation against bit errors due to the significant separation of the modulation phases compared to DQPSK modulation. In fact, the constellation diagram of the two modulations [48] shows that in DBPSK, there is a $180^{\circ}$ separation between each two transmitted symbols, for only $45^{\circ}$ between the four symbols in DQPSK. Thus, much more packet loss can occur with DQPSK modulation if the transmission channel is subject to a high level of interference (in the case of high data rates).

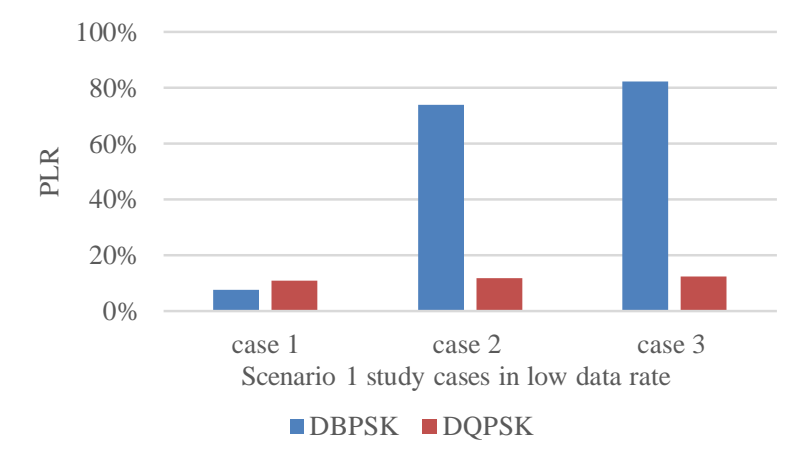

Fig. 3. Scenario 1: PLR (R-BAN) for DBPSK and DQPSK Modulation Schemes at Low Data Rate (3kbps).

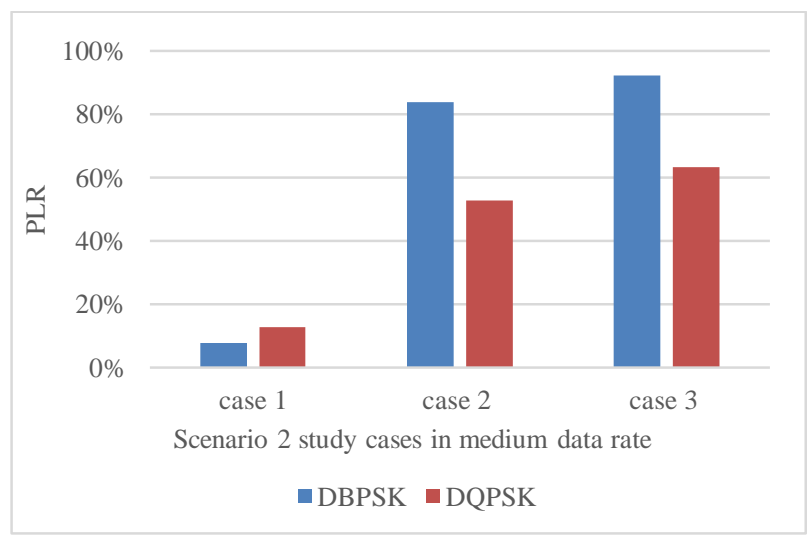

Fig. 4. Scenario 1: PLR (R-BAN) for DBPSK and DQPSK Modulation Schemes at Medium Data Rate Transmission (44 kbps).

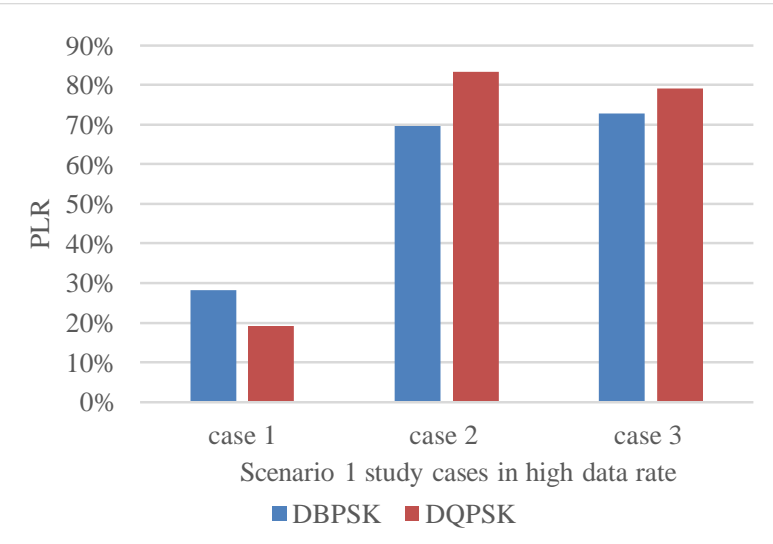

Fig. 5. Scenario 1: PLR (R-BAN) for DBPSK and DQPSK Modulation Schemes for High Data Rate Transmission (72 kbps).

B. Scenario 2: Influence of DBPSK and DQPSK Modulation Schemes on the Transmission Performance (PLR) of R$B A N$, Depending on the Number of Nodes in I-BAN

In the present study, the scenario 2 described earlier in Section III is simulated, by studying for each of the two modulation schemes (DBPSK and DQPSK), the impact of the number of I-BAN nodes on transmission performance of RBAN in the three transmission data rates: low, medium and high data rate. 
Fig. 6, 7 and 8 successively present the packet loss rate (PLR) of R-BAN, at low medium and high data rate respectively and for different values of the number of nodes $\mathrm{N}$ in I-BAN (Case 1: $\mathrm{N}=2$, case 2: $\mathrm{N}=5$, case 3: $\mathrm{N}=10$, case 4: $\mathrm{N}=15$ and case $5: \mathrm{N}=21$ ).

An initial analysis of Fig. 6, 7 and 8 shows that, at low data rates, R-BAN transmission performance (PLR) in DQPSK modulation has a relatively correct PLR $(\approx 10$ to $12 \%)$ and is practically unaffected by variations in the number of nodes of I-BAN. This is certainly not the case with DBPSK modulation which, under the same study conditions, clearly shows poor performance and seems to worsen further with a larger number of I-BAN nodes. This performance degradation obtained with DBPSK modulation is due once again to the sensitivity of the receiver which, in DBPSK, allows even weak signals from IBAN to be received.

For medium and high data rates, and when DQPSK modulation is used, PLR deteriorates significantly, especially at high data rates. This performance degradation with DQPSK is mainly related to the weakness of this modulation scheme in front of bit error occurrences, which are much more frequent in the case of strong interference in the R-BAN transmission channel.

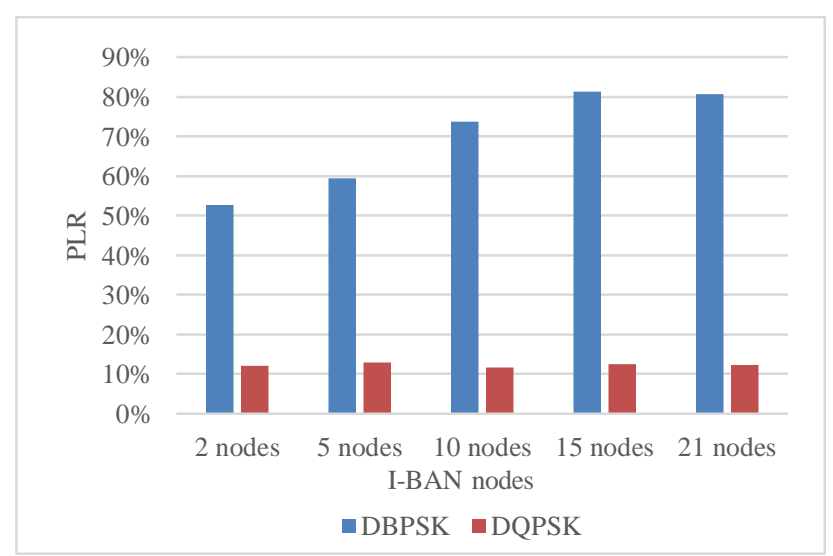

Fig. 6. Scenario 2: PLR (R-BAN) Evaluation, at Low Data Rates (3kbps) for DBPSK and DQPSK as a Function of the Number of nodes in I-BAN.

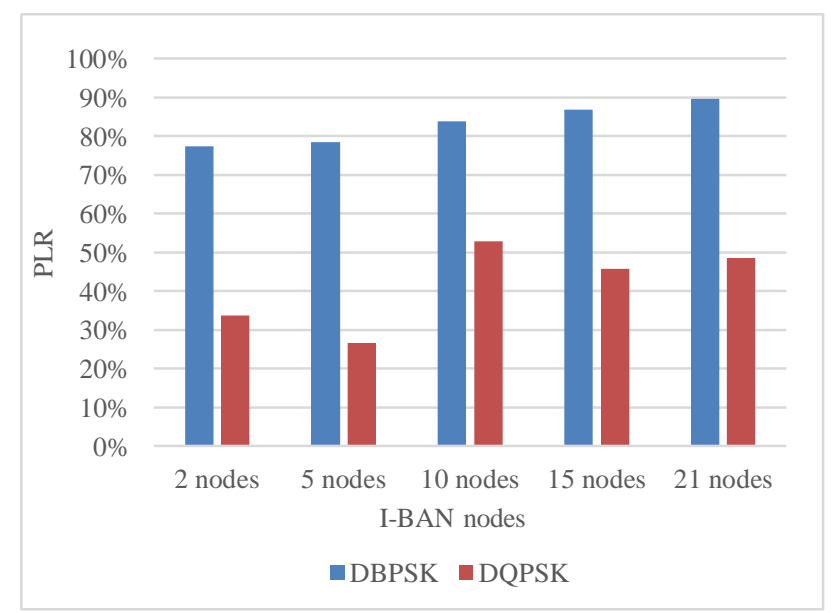

Fig. 7. Scenario 2: PLR (R-BAN) Evaluation, at Medium Data Rates (44kbps) for DBPSK and DQPSK as a Function of the Number of Nodes in IBAN.

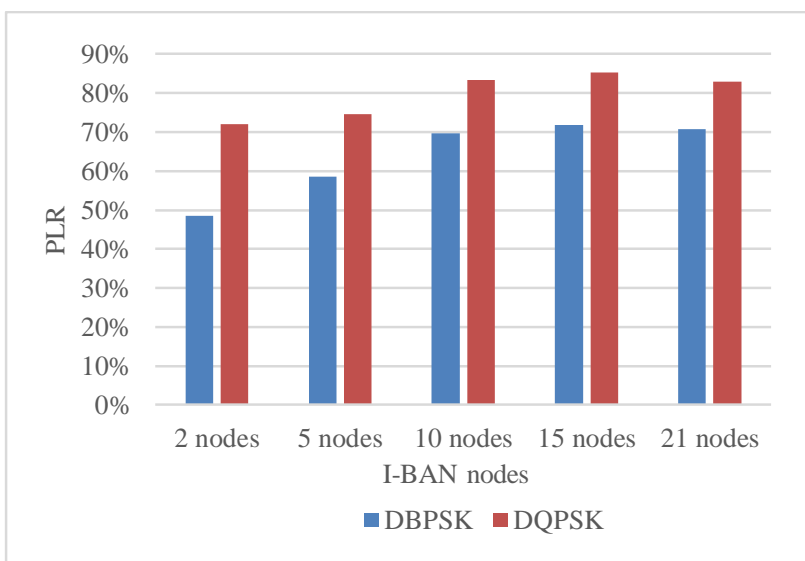

Fig. 8. Scenario 2: PLR (R-BAN) Evaluation, at High Data Rates (72kbps) for DBPSK and DQPSK as a Function of the Number of Nodes in I-BAN.

C. Scenario 3: Influence of the DBPSK and DQPSK Modulation Schemes on the Transmission Performance (PLR) of R-BAN, as a Function of Traffic Payload at IBAN Level

To present the effect of traffic payload of I-BAN on mutual interference, the packet loss rate reached, at low, medium and high data rates is shown in Fig. 9, 10 and 11, respectively, in the cases where I-BAN transmits its packets with payloads of 10,100 and 200 Bytes. The reference BAN payload is always maintained at 100 Bytes.

The results of this scenario show, once again, as for the two previous scenarios, that the traffic payload of I-BAN traffic has an impact on the PLR received at R-BAN. Moreover, for the same reasons of receiver sensitivity discussed above, the PLR reached at low and medium data rates (Fig. 9 and 10), with DQPSK modulation, is better than in DBPSK. However, at high data rates (Fig. 11), this behavior is inverted in favor of DBPSK modulation when the interference level increases (high data rate).

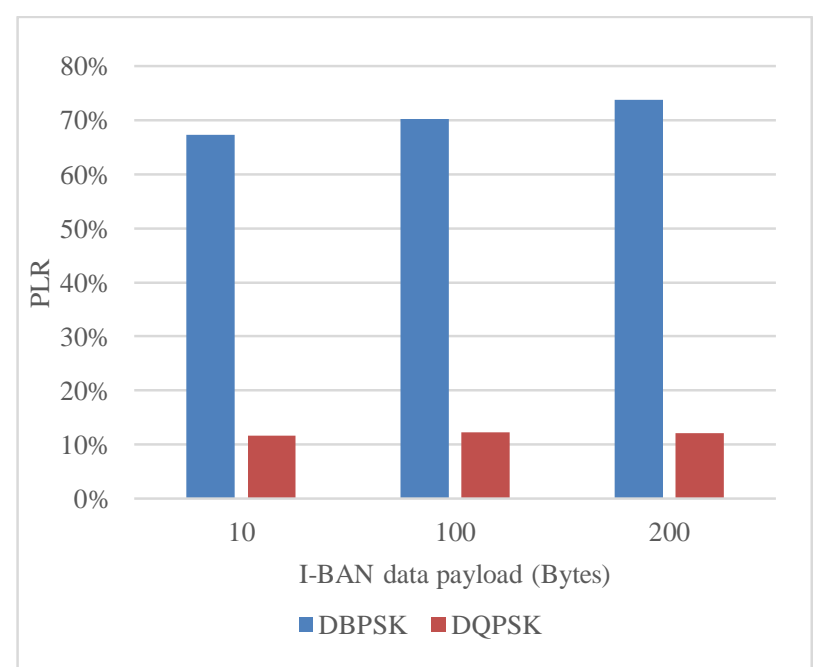

Fig. 9. Scenario 3: PLR (R-BAN) Evaluation at Low Data Rate (3kbps) for DBPSK and DQPSK as a Function of I-BAN Traffic Payload. 


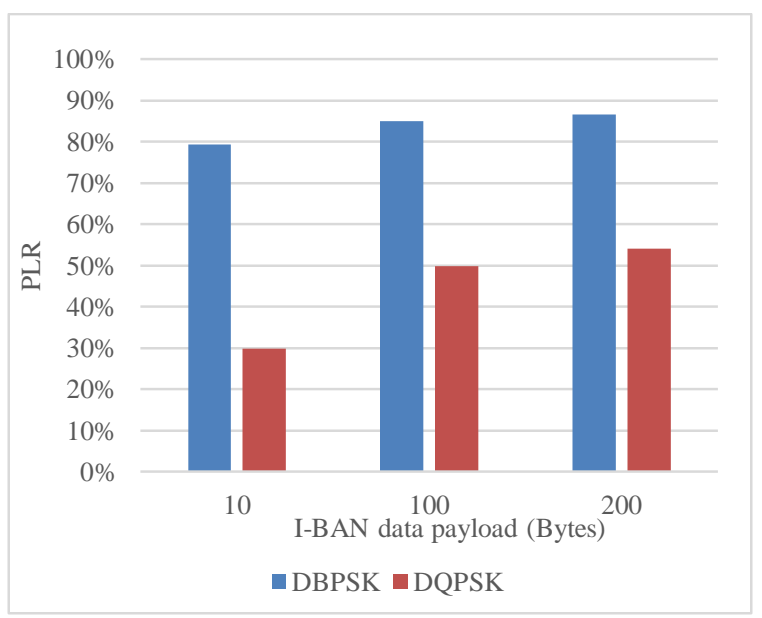

Fig. 10. Scenario 3: PLR (R-BAN) Evaluation at Medium Data Rate (44kbps) for DBPSK and DQPSK as a Function of I-BAN Traffic Payload.

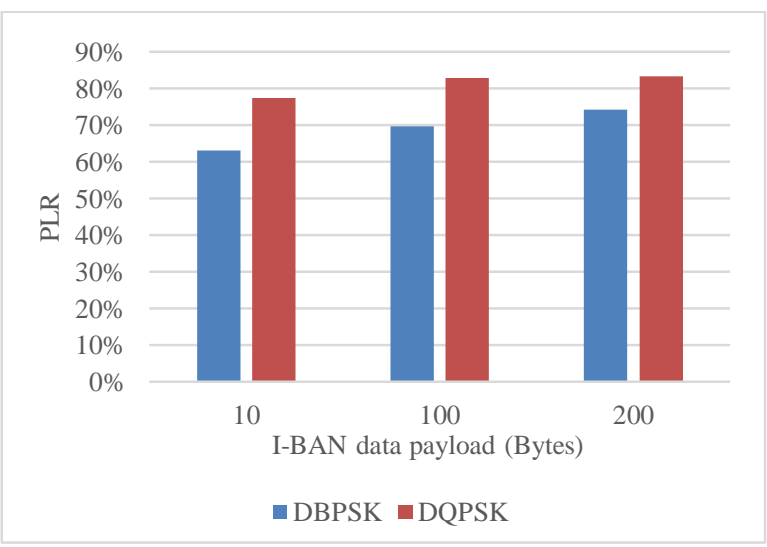

Fig. 11. Scenario 3: PLR (R-BAN) Evaluation at High Data Rate (72kbps) for DBPSK and DQPSK as a Function of I-BAN Traffic Payload.

The obtained results show that in the absence of adjacent BANs, the choice of DBPSK modulation in low data rate onbody medical applications such as: body temperature sampling (120 bps) or blood pressure measurement ( $<10 \mathrm{bps})$, and in medium data rate applications such as: EEG signal monitoring (43.2 kbps) or pulse oximetry (32 kbps), guarantees better PLR performance compared to DQPSK modulation. However, in these applications, switching to DQPSK modulation in the case of mutual interference is necessary in order to reduce the packet loss rate, without increasing the transmission power, which will reduce the energy efficiency of the nodes.

For high data rate on-body medical applications, such as ECG monitoring (71 kbps), the use of DQPSK modulation may be a good choice only in an ideal condition, where no other BAN is nearby. Nevertheless, switching to DBPSK modulation is required in the case of interference from other adjacent BANs to lower the packet loss rate, due to its robustness to maintain an acceptable link quality even when the interference level becomes more pronounced.

\section{CONClusion}

This paper investigated and analyzed the impact of the choice of DBPSK and DQPSK modulation schemes (described in the $2.4 \mathrm{GHz}$ narrow band of the IEEE 802.15.6 standard), on the transmission performance of a BAN in a mutual interference environment. Performance is evaluated by using Packet Loss Rate (PLR) at various data rate levels. In this context, three study scenarios were examined, which investigate the effects of inter-BAN distance, number of nodes and traffic payload of the interferer BAN respectively, on the performance of a reference BAN.

The numerical study carried out on the three scenarios was able to show the impact of the three cited parameters on the studied performance for each of the two considered modulation schemes. At low and medium data rates, DQPSK modulation has shown better results in terms of PLR, however at high data rates the BAN transmission performance degrades for both modulation schemes, but DBPSK remains relatively more efficient.

The battery power supplying the nodes in a WBAN can deteriorate due to co-channel interference, resulting in a decrease in SINR which will cause throughput degradation. Therefore, Green communication based energy efficient techniques for co-channel interference BANs, is planned as a perspective to finalize this work.

\section{REFERENCES}

[1] ES-SAQY et al., "Very Low Phase Noise Voltage Controlled Oscillator for 5G mm-wave Communication Systems," 20201 st International Conference on Innovative Research in Applied Science, Engineering and Technology (IRASET), Meknes, Morocco, 2020, pp. 1-4, doi: 10.1109/IRASET48871.2020.9092005.

[2] R. Negra, I. Jemili, and A. Belghith, "Wireless Body Area Networks: Applications and Technologies," Procedia Comput. Sci., vol. 83, no. May, pp. 1274-1281, 2016, doi: 10.1016/j.procs.2016.04.266.

[3] T. Hayajneh, G. Almashaqbeh, S. Ullah, and A. V. Vasilakos, A survey of wireless technologies coexistence in WBAN: analysis and open research issues, vol. 20, no. 8. 2014, doi: 10.1007/s1 1276-014-0736-8

[4] M. BOUMAIZ et al., "Energy harvesting based WBANs: EH optimization methods," Procedia Comput. Sci., vol. 151, pp. 1040-1045, 2019, doi: 10.1016/j.procs.2019.04.147.

[5] L. A. N. Man, S. Committee, and I. Computer, IEEE Standard for Local and metropolitan area networks $Y$ Part 15.6: Wireless Body Area Networks IEEE Computer Society, no. February. 2012, doi: 10.1109/IEEESTD.2012.6161600.

[6] S. Movassaghi, M. Abolhasan, J. Lipman, D. Smith, and A. Jamalipour, "Wireless Body Area Networks: A Survey," Ieee Commun. Surv. Tutorials, vol. 16, no. 3, pp. 1658-1686, 2014, doi: 10.1109/surv.2013.121313.00064.

[7] R. Cavallari, F. Martelli, R. Rosini, C. Buratti, and R. Verdone, "A survey on wireless body area networks: Technologies and design challenges," IEEE Commun. Surv. Tutorials, vol. 16, no. 3, pp. 16351657, 2014, doi: 10.1109/SURV.2014.012214.00007.

[8] P. Mathew, L. Augustine, and D. Selvakumar, "Hardware Implementation of NB PHY Baseband Transceiver for IEEE 802 . 15 .6 WBAN," pp. 64-71, 2014 , doi: 10.1109/MedCom.2014.7005977

[9] Y. Wang, Q. Wang, "Evaluating the IEEE 802.15.6 2.4GHz WBAN Proposal on Medical Multi-Parameter Monitoring under WiFi/Bluetooth Interference", International Journal of E-Health and Medical Communications, vol. 2, no. 3, July 2011, doi: 10.4018/jehmc. 2011070103.

[10] Wang Z., Jiang H. and Chen H., "WBAN Transceiver Design" In: CMOS IC Design for Wireless Medical and Health Care. Springer, New York, NY, pp 73-120,2004, doi: 10.1007/978-1-4614-9503-1_4.

[11] W. Yang and K. Sayrafian-pour, "Interference Mitigation for Body Area Networks," pp. 2193-2197, 2011, doi: 10.1109/PIMRC.2011.6139905

[12] M. Boumaiz and M. Fattah, "The impact of distance between neighboring WBANs," 2019 7th Mediterr. Congr. Telecommun., pp. 1-4, 2019, doi: 10.1109/CMT.2019.8931341 
[13] P. R. Grassi, V. Rana, I. Beretta, and D. Sciuto, "2012 Ninth International Conference on Wearable and Implantable Body Sensor Networks B 2 IRS : a Technique to Reduce BAN-BAN Interferences in Wireless Sensor Networks," 2012, doi: 10.1109/BSN.2012.30.

[14] S. Movassaghi, M. Abolhasan, and D. Smith, "Interference Mitigation in WBANS : Challenges and Existing solutions," Work. Adv. Real-time Inf. Networks, 2013, doi: 10.5130/aaa.b

[15] M. Barbi, K. Sayrafian and M. Alasti, "Low complexity adaptive schemes for energy detection threshold in the IEEE 802.15.6 CSMA/CA," 2016 IEEE Conference on Standards for Communications and Networking (CSCN), Berlin, 2016, pp. 1-6, doi: 10.1109/CSCN.2016.7785182.

[16] I. Dotlic, "Interference performance of IEEE 802 . 15 . 6 Impulse - Radio Ultra - Wideband Physical Layer," no. September 2011, 2014, doi: 10.1109/PIMRC.2011.6139895.

[17] D. M. Davenport, B. Deb, and F. J. Ross, "Wireless Propagation and Coexistence of Medical Body Sensor Networks for Ambulatory Patient Monitoring," pp. 8-12, 2009, doi: 10.1109/BSN.2009.8.

[18] L. W. Hanlen, D. Miniutti, D. Smith, D. Rodda, and B. Gilbert, "CoChannel Interference in Body Area Networks with Indoor Measurements at $2.4 \mathrm{GHz}$ : Distance-to-Interferer is a Poor Estimate of Received Interference Power," pp. 113-125, 2010,doi:10.1007/s10776-010-0123-z.

[19] X. Wang and L. Cai, "Interference Analysis of Co-existing Wireless Body Area Networks," pp. 0-4, 2011, doi: 10.1109/GLOCOM. 2011.6133624

[20] A. Zhang, D. B. Smith, D. Miniutti, L. W. Hanlen, D. Rodda, and B. Gilbert, "Performance of Piconet Co-existence Schemes in Wireless Body Area Networks," 2010, doi: 10.1109/WCNC.2010.5506746

[21] E. K. Sungkwan, Y. Taeshik, and C. Kang, "Asynchronous inter-network interference avoidance for wireless body area networks," pp. 562-579, 2013, doi: 10.1007/s11227-012-0840-4.

[22] Kim, S., Kim, S., Kim, J.W., Eom, D.S.: A beacon interval shifting scheme for interference mitigation in body area networks. Sensors 12(8), 10,930-10,946 (2012).

[23] L. Wang, C. Goursaud, N. Nikaein, L. Cottatellucci, and J. Gorce, "Coexisting Body Area Networks," vol. 12, no. 1, pp. 123-133, 2013, , doi: 10.1109/TWC.2012.120412.112073

[24] J. Mahapatro, S. Misra, S. Member, M. Manjunatha, and N. Islam, "Interference Mitigation Between WBAN Equipped Patients," 2012 , doi: 10.1109/WOCN.2012.6331909

[25] T. T. T. Le and S. Moh, "Link Scheduling Algorithm with Interference Prediction for Multiple Mobile WBANs," 2017, doi: 10.3390/s17102231.

[26] Z. Xie et al., "An Optimal Backoff Time-Based Internetwork Interference Mitigation Method in Wireless Body Area Network," vol. 2020, 2020, , doi: $10.1155 / 2020 / 4365191$

[27] J. Han, H. Kim, J. Bang, and Y. Lee, "Interference Mitigation in IEEE 802.15. 4 Networks," no. 1, 2011, doi: 10.1109/GLOCOM.2011.6134377

[28] W. Lee and S. H. Rhee, "Channel Management Protocol for Wireless Body Area Networks,” pp. 494-497, 2009, doi: 10.1007/978-3-64204492-2_61

[29] Han, S., Lee, S., Lee, S., Kim, Y.: Coexistence perfor mance evaluation of ieee 802.15. 4 under ieee $802.11 \mathrm{~b}$ in terference in fading channels. In: Proc of IEEE PIMRC, pp. 1-5 (2007)

[30] G. Wu, J. Ren, F. Xia, L. Yao, and Z. Xu, "DISG : Decentralized InterUser Interference Suppression in Body Sensor Networks with Noncooperative Game,” 2010, doi: 10.1109/UIC-ATC.2010.6.

[31] K. Ghanem and P. S. Hall, "Interference cancellation using CDMA multiuser detectors for on-body channels," pp. 2152-2156, 2009, doi: 10.1109/PIMRC.2009.5450218

[32] J. N. Bae, Y. H. Choi, J. Y. Kim, J. W. Kwon, and D. I. Kim, "Efficient Interference Cancellation Scheme for Wireless Body Area Network," vol. 13, no. 2, pp. 167-174, 2011, doi: 0.1109/JCN.2011.6157416

[33] W. Yang and K. Sayrafian-Pour, "A DS-CDMA Interference Cancellation technique for body area networks," 21st Annual IEEE International Symposium on Personal, Indoor and Mobile Radio Communications, Instanbul, 2010, pp. 752-756, doi: 10.1109/PIMRC. 2010.5671929
[34] A. Tawfiq, J. Abouei and K. N. Plataniotis, "Cyclic orthogonal codes in CDMA-based asynchronous Wireless Body Area Networks," 2012 IEEE International Conference on Acoustics, Speech and Signal Processing (ICASSP), Kyoto, 2012, pp. 1593-1596, doi: 10.1109/ICASSP. 2012.6288198

[35] W.-B. Yang and K. Sayrafian-Pour, "Interference Mitigation Using Adaptive Schemes in Body Area Networks," Int. J. Wirel. Inf. Networks, vol. 19, no. 3, pp. 193-200, 2012, doi: 10.1007/s10776-012-0192-2.

[36] C. M. Liang, N. B. Priyantha, J. Liu, and A. Terzis, "Surviving Wi-Fi Interference in Low Power ZigBee Networks," pp. 309-322, 2010, doi: $10.1145 / 1869983.1870014$.

[37] J. Hou, B. Chang, D. Cho, and M. Gerla, "Minimizing 802.11 Interference on Zigbee Medical Sensors," 2009, doi: 10.4108 /ICST.BODYNETS2009.6029

[38] J. Huang, G. Xing, G. Zhou, and R. Zhou, "Beyond Co-existence: Exploiting WiFi White Space for ZigBee Performance Assurance," pp. 305-314, 2010, doi: 10.1109/ICNP.2010.5762779

[39] K. Lee, C. Chae, T. Sung, and J. Kang, "Cognitive Beamforming Based Smart Metering for Coexistence with Wireless Local Area Networks," vol. 14, no. 6, pp. 619-628, 2012, doi: 10.1109/JCN.2012.00028

[40] Y. Wang, Q. Wang, Z. Zeng, G. Zheng, and R. Zheng, "WiCop: Engineering WiFi Temporal White-Spaces for Safe Operations of Wireless Body Area Networks in Medical Applications," pp. 170-179, 2011, doi: 10.1109/RTSS.2011.23.

[41] B. De Silva, A. Natarajan, and M. Motani, "Inter-User Interference in Body Sensor Networks : Preliminary Investigation and an InfrastructureBased Solution,” pp. 3-8, 2009, doi: 10.1109/BSN.2009.36.

[42] W. Sun, Y. Ge, W. Wong, and S. Member, "A Lightweight Inter-User Interference Mitigation Method in Body Sensor Networks," pp. 34-40, 2012, doi:1 0.1109/WiMOB.2012.6379098

[43] M. Boumaiz et al., "The impact of transmission power on the performance of a WBAN prone to mutual interference," 2019 Int. Conf. Syst. Collab. Big Data, Internet Things Secur., pp. 1-4, 2019, doi: 10.1109/SysCoBIoTS48768.2019.9028035.

[44] S. Ullah et al., "A comprehensive survey of wireless body area networks on PHY, MAC, and network layers solutions," J. Med. Syst., vol. 36, no. 3, pp. 1065-1094, 2012, doi: 10.1007/s10916-010-9571-3.

[45] F. Di Franco, C. Tachtatzis, R. C. Atkinson, I. Tinnirello, and I. A. Glover, "Channel estimation and transmit power control in wireless body area networks," no. May 2013, pp. 11-19, 2015, doi: 10.1049/ietwss.2013.0070.

[46] K. Takizawa, Channel model for wearable WBAN, no. July. 2008. [online]. Available: https://mentor.ieee.org/802.15/documents

[47] M. Boumaiz, M. E. L. Ghazi, S. Mazer, M. E. L. Bekkali, A. Bouayad, and M. Fattah, "Performance analysis of DQPSK and DBPSK modulation schemes for a scheduled access phase based Wireless Body Area Network," 2018 9th Int. Symp. Signal, Image, Video Commun., pp. 163-167, 2018, doi: 10.1109/ISIVC.2018.8709232

[48] Ningdalli, Ashwini and Dr. B. K. Sujatha. "FPGA Implementation of PI/2 BPSK and PI/4 QPSK for IEEE 802.15.6 WBAN Standard.” (2015),

[49] P. S. Hall, "Antennas challenges for body centric communications," Conf. Proc. - 2007 IEEE Int. Work. Antenna Technol. Small Smart Antennas Metamaterials Appl. iWAT 2007, no. 2, pp. 41-44, 2007, doi: 10.1109/IWAT.2007.370076.

[50] S. L. Cotton, R. D. Errico, and C. Oestges, "A review of radio channel models for body centric communications," pp. 371-388, 2014, doi: 10.1002/2013RS005319.Received.

[51] K. Y. Yazdandoost, Channel Modeling Subcommittee Report. 2010. [online]. Available: https://mentor.ieee.org/802.15/documents

[52] A. Chi et al., "A 1 V 5 mA Multimode IEEE 802.15.6/Bluetooth LowEnergy WBAN Transceiver for Biotelemetry Applications," vol. 48, no. 1, pp. 186-198, 2013. doi: https://doi.org/10.1109/JSSC.2012.2221215

[53] A. Boulis, "Castalia A simulator for Wireless Sensor Networks and Body Area Networks - User's Manual,” Version 3.0, no. March, p. 79, 2010. 